\title{
Laparoscopic ischaemic conditioning of the gastric conduit prior to a hybrid mckeown oesophagectomy may not decrease the risk of anastomotic leak
}

\author{
Nader Hanna, Zuhaib M. Mir, Erin Williams, Shaila J. Merchant, Boris Zevin, Wiley Chung \\ Department of Surgery, Queen's University, Kingston, Canada
}

Videosurgery Miniinv 2021; 16 (4): 669-677

DOI: https://doi.org/10.5114/wiitm.2021.105529

\begin{abstract}
Introduction: Morbidity associated with anastomotic leak after oesophagectomy is significant. Techniques to reduce this risk include ischaemic conditioning of the gastric conduit prior to oesophagectomy.

Aim: To quantify the rate of anastomotic leak after a hybrid minimally invasive McKeown oesophagectomy preceded by laparoscopic gastric devascularization (LGD).

Material and methods: We identified patients who had undergone neoadjuvant chemoradiotherapy followed by LGD and McKeown oesophagectomy and conducted a retrospective case series. The primary outcome was anastomotic leak, and secondary outcomes included common post-operative complications within 30 days.

Results: Eleven patients were identified. Seventy-three per cent were male, and 7 of 11 patients were age $70+$ years. 91\% of tumours were located in the lower oesophagus or gastroesophageal junction (GEJ), and $72 \%$ of the series had clinical stage of II-III. The median ischaemic conditioning time was 15 days. Eighteen per cent of patients developed an anastomotic leak, and all were managed non-operatively. One patient developed an anastomotic stricture. Three patients developed pneumonia. Three patients suffered wound infection at the site of the neck incision. One had respiratory failure requiring ventilator support. None required reoperation or readmission. There were no mortalities following either operation.

Conclusions: Laparoscopic ischaemic conditioning via LGD prior to a hybrid McKeown oesophagectomy for malignancy was associated with a leak rate similar to previously published data for a McKeown oesophagectomy without prior LGD.
\end{abstract}

Key words: McKeown oesophagectomy, anastomotic leak, laparoscopic gastric devascularization, ischaemic conditioning, hybrid.

\section{Introduction}

Gastric interposition is the most common method of reconstruction following oesophagectomy in end-stage benign or malignant oesophageal disease. There are several surgical approaches to oesophagectomy, each with distinct complication profiles; however, the most concerning complication that is common to all approaches is that of gastric conduit ischaemia and oesophagogastric anas- tomotic leak. The incidence of anastomotic leak can be up to $13 \%$ for an Ivor-Lewis oesophagectomy and $20 \%$ for a McKeown oesophagectomy [1, 2]. Anastomotic leaks following oesophagectomy are associated with significant morbidity and mortality, and some authors have reported a decrease in quality of life and worse oncological outcomes compared to those who did not experience a leak $[3,4]$. 
Several studies have postulated that ischaemic changes around the gastric fundus as a consequence of creation of the conduit is the biggest risk factor for anastomotic leak [5-10]. Sonographic studies have shown impaired perfusion at the tip of the conduit following ligation of the gastric vessels, despite preservation of the right gastroepiploic artery [11-14]. Consequently, methods to reduce the risk of anastomotic leak have been proposed. One such technique is ischaemic conditioning of the stomach, where several weeks prior to the oesophagectomy, vessels supplying the stomach are either ligated surgically or embolized percutaneously, leaving the conduit reliant solely on the right gastroepiploic artery for perfusion $[15,16]$. The extent of devascularization is variable - some promote ligation of only the left gastric vessels, while others advocate a more extensive procedure including the coronary vein, the left gastroepiploic artery, and the short gastric vessels [17-20]. The stomach is then used as a replacement for the resected oesophagus, to restore gastrointestinal tract continuity.

Human and animal studies [21] have demonstrated that gastric devascularization prior to delayed resection and reconstruction allows for neovascularization of the gastric conduit, which may reduce rates of anastomotic leak and stricture. A previous study showed that ischaemic conditioning of the stomach by laparoscopic gastric devascularization (LGD) was associated with a reduction of the intrathoracic anastomotic leak rate to as low as 3\% for an Ivor-Lewis oesophagectomy [22]. To date, there have been no studies investigating the outcomes of this technique for a hybrid minimally invasive (thoracoscopy-laparotomy) Mckeown oesophagectomy.

\section{Aim}

We aimed to describe our surgical technique, with LGD performed up to 25 days prior to a hybrid Mckeown oesophagectomy, and the post-operative anastomotic leak rate associated with this.

\section{Material and methods}

\section{Study population}

We performed a retrospective review of patients who underwent LGD prior to a hybrid minimally invasive Mckeown oesophagectomy between Feb- ruary 2017 and February 2018 at a tertiary referral centre. All patients had malignant disease and received neoadjuvant Carboplatin and Paclitaxel with concurrent radiotherapy. Data on patient and disease characteristics and post-operative outcomes were collected. Ethical approval was obtained via the Health Sciences Research Ethics Board at Queen's University, Kingston, Canada.

The primary outcome was any evidence of anastomotic leak (i.e. contained, free) at the oesophagogastric anastomosis identified on oesophagram. Secondary outcomes were anastomotic stricture requiring endoscopic dilation, post-operative cardiorespiratory complications (i.e. pneumonia, empyema, respiratory failure, atrial fibrillation, cardiac arrest), surgical site infections of any severity, venous thromboembolic events (i.e. pulmonary embolism, deep vein thrombosis), reoperation, and inpatient mortality. These post-operative complications were selected because they are commonly reported in the oesophagectomy literature and are of clinical relevance to surgeons performing oesophagectomy.

\section{Laparoscopic gastric devascularization}

The patient is positioned supine in the split leg position and undergoes general anaesthesia after administration of antibiotic and venous thromboembolism prophylaxis. A 12-mm optical trocar is inserted in the left upper quadrant followed by a $5-\mathrm{mm}$ camera port placed $17 \mathrm{~mm}$ caudal to the xiphoid process just left of the midline. Three additional 5-mm ports are then placed: one in the epigastrium, one in the left flank, and one in the right mid abdomen. Here, diagnostic laparoscopy is conducted to rule out metastatic disease, looking specifically at the liver, stomach, small bowel, omentum, and peritoneum.

A Nathanson ${ }^{\circledR}$ retractor is placed through the epigastric port to elevate the left lobe of the liver. Attention is turned to the greater curvature of the stomach where the short gastric vessels are divided using an energy device, from the inferior pole of the spleen to the base of the left crus. The posterior attachments of the stomach are divided to facilitate mobilization. This exposes the left gastric artery and coronary vein, which are divided with a vascular endoscopic stapler. The gastrocolic omentum is divided to ligate the left gastroepiploic artery while taking care to preserve the right gastroepiploic artery and its branches. 


\section{Hybrid minimally invasive McKeown oesophagectomy}

All oesophagectomies begin with thoracoscopy. The patient is first placed in the left lateral decubitus position with single-lung ventilation. A $12-\mathrm{mm}$ camera port is placed in the $7^{\text {th }}$ intercostal space in the right posterior axillary line, followed by three $5-\mathrm{mm}$ ports and a 3-cm utility port. Inspection of the right thoracic cavity to rule out metastatic disease is performed prior to mobilization of the oesophagus. The oesophagus and surrounding lymphatic tissue are then mobilized from the level of the thoracic inlet to the oesophageal hiatus. The azygous vein is isolated and divided with a vascular endoscopic stapler. The thoracic duct is prophylactically ligated with a clip. Two chest tubes are placed, one in the posterior-apical space and one in the subpulmonic space.

The patient is repositioned supine, and an upper midline laparotomy is carried out. The right gastroepiploic vascular arcade is identified, protected, and mobilized to its origin. The hepatic flexure of the colon is mobilized to facilitate full Kocherization of the duodenum. The pars flaccida is divided and all posterior attachments of the stomach to the pancreas are divided. Circumferential hiatal dissection is then performed. All periesophageal lymphatic tissue and lymph nodes along the left gastric vessel are included in the surgical specimen.

Following this, a left neck incision anterior to the sternocleidomastoid is performed to expose the cervical oesophagus, avoiding injury to the left recurrent laryngeal nerve. The oesophagus is divided and delivered through the hiatus into the abdomen. The gastric conduit is fashioned using serial firings of an endoscopic stapler and brought up past the posterior mediastinum and into the left neck. Once the proximal oesophageal and distal gastric margins are confirmed to be negative on frozen section, the oesophagogastric anastomosis is performed either with an endoscopic stapler or in a handsewn fashion, which is left to the discretion of the operating surgeon. Next, a leak test using endoscopic insufflation is performed to ensure anastomotic integrity.

A feeding jejunostomy tube is placed prior to laparotomy closure. A Penrose drain is inserted in the left neck via a separate incision. Postoperatively, patients are transferred to the intensive care unit and extubated the following morning. An oesophagram is performed on postoperative day 7 to assess for anastomotic leak. A clear fluid diet is initiated and progressed to a full fluid diet that is maintained until the first postoperative clinic visit.

\section{Statistical analysis}

All data were collected and analysed using Microsoft Excel (version 16.12 2018). Descriptive data are presented as proportion (\%), median (range), and mean ( \pm standard deviation) where appropriate.

\section{Results}

\section{Study population}

In the period between February 2017 to February 2018 a total of 11 patients met the inclusion criteria. Table I summarizes the patient and disease characteristics for the study population. The mean age was $68 \pm 8.8$ years, and 8 (73\%) were male. Two (18\%) patients were lifelong non-smokers, $8(73 \%)$ were ex-smokers, and 1 (9\%) patient admitting to actively smoking. The median body mass index (BMI) was $26(21-34) \mathrm{kg} / \mathrm{m}^{2}$. No patients had prior thoracic surgery. The most common presenting complaint was dysphagia, with $8(73 \%)$ patients being referred to the outpatient clinic for this reason, 2 patients experienced melena, and 1 haematemesis. Three (27\%) patients had previously documented diagnosis of Barrett's oesophagus. All 11 patients had a pre-operative $\mathrm{FEV}_{1}$ of greater than $80 \%$. Two (18\%) patients were deemed American Society of Anesthesiologists (ASA) class 4 during their pre-operative anaesthetic assessment, while the remaining $9(82 \%)$ were classified as ASA 3. Four (36\%) patients experienced a complete pathological response on final surgical pathology, with $2(18 \%)$ achieving significant cancer down-staging.

Table II summarizes the operative factors. Metastatic disease was not found in any patient during the LGD. The mean LGD operative time was $65 \pm 12$ min. All patients were discharged home the same day. The median ischaemic conditioning time was 15 (8-25) days. The only complication that occurred during LGD was chyloperitoneum in 1 patient, which was managed by ligation during subsequent oesophagectomy. The median oesophagectomy operative time was 448 (406-800) min. Eight patients had a stapled anastomosis (in either an end-to-end or side-to-side fashion) and 3 had a hand-sewn anastomosis. One patient suffered splenic laceration during the laparotomy portion of the oesophagec- 
Table I. Demographic and disease characteristics of patients with oesophageal cancer undergoing neoadjuvant chemoradiation, laparoscopic gastric devascularization followed by McKeown esophagectomy, categorized by the detection of anastomotic leak on oesophagram

\begin{tabular}{|c|c|c|c|}
\hline Characteristic & $\begin{array}{c}\text { Total }(n=11) \\
n(\%)\end{array}$ & $\begin{array}{c}\text { Leak }(n=2) \\
n\end{array}$ & $\begin{array}{c}\text { No leak }(n=9) \\
n\end{array}$ \\
\hline \multicolumn{4}{|l|}{ Age [years]: } \\
\hline $50-59$ & $3(27)$ & 1 & 2 \\
\hline $60-69$ & $1(9)$ & 1 & 0 \\
\hline $70-79$ & $6(55)$ & 0 & 6 \\
\hline $80+$ & $1(9)$ & 0 & 1 \\
\hline \multicolumn{4}{|l|}{ Sex: } \\
\hline Male & $8(73)$ & 2 & 6 \\
\hline Female & $3(27)$ & 0 & 3 \\
\hline \multicolumn{4}{|l|}{ BMI $\left[\mathrm{kg} / \mathrm{m}^{2}\right]$ : } \\
\hline $20-24$ & $4(36)$ & 1 & 3 \\
\hline $25-29$ & $6(55)$ & 1 & 5 \\
\hline $30-34$ & $1(9)$ & 0 & 1 \\
\hline \multicolumn{4}{|l|}{ Smoker: } \\
\hline Current & $1(9)$ & 0 & 1 \\
\hline Previous & $8(73)$ & 2 & 6 \\
\hline Never & $2(18)$ & 0 & 2 \\
\hline \multicolumn{4}{|l|}{ ASA grade: } \\
\hline III & $8(73)$ & 2 & 7 \\
\hline IV & $3(27)$ & 0 & 2 \\
\hline \multicolumn{4}{|l|}{ Cardiac comorbidity: } \\
\hline None & $4(36)$ & 1 & 3 \\
\hline Hypertension & $3(27)$ & 1 & 2 \\
\hline Valve disease & $3(27)$ & 0 & 3 \\
\hline Atrial fibrillation & $1(9)$ & 0 & 1 \\
\hline \multicolumn{4}{|l|}{ Diabetes mellitus type 2: } \\
\hline No & $9(82)$ & 2 & 7 \\
\hline Yes & $2(18)$ & 0 & 2 \\
\hline \multicolumn{4}{|l|}{ COPD: } \\
\hline No & $8(73)$ & 2 & 6 \\
\hline Yes & $3(27)$ & 0 & 3 \\
\hline \multicolumn{4}{|l|}{$\mathrm{FEV}_{1}$ predicted: } \\
\hline $80-90 \%$ & $4(36)$ & 1 & 3 \\
\hline $90-100 \%$ & $7(64)$ & 1 & 6 \\
\hline \multicolumn{4}{|l|}{ Tumour location: } \\
\hline Upper oesophagus & $0(0)$ & 0 & 0 \\
\hline Middle oesophagus & $1(9)$ & 0 & 1 \\
\hline Lower oesophagus and GEJ & $10(91)$ & 2 & 8 \\
\hline \multicolumn{4}{|l|}{ Clinical stage: } \\
\hline I & $3(27)$ & 0 & 3 \\
\hline II & $4(36)$ & 1 & 3 \\
\hline III & $4(36)$ & 1 & 3 \\
\hline
\end{tabular}

BMI - body mass index, ASA - American Society of Anesthesiologists, COPD - chronic obstructive pulmonary disease, FEV - forced expiratory volume, GEJ-gastroesophageal junction. 
Table II. Perioperative factors of patients undergoing laparoscopic gastric devascularization and McKeown oesophagectomy, categorized by the detection of anastomotic leak on oesophagram

\begin{tabular}{|c|c|c|c|}
\hline Variable & $\begin{array}{l}\text { Total }(n=11) \\
n \text { or }(\%)\end{array}$ & $\begin{array}{c}\text { Leak }(n=2) \\
n\end{array}$ & $\begin{array}{c}\text { No leak }(n=9) \\
n\end{array}$ \\
\hline \multicolumn{4}{|l|}{ Ischaemic conditioning time [days]: } \\
\hline Median & 15 & 16 & 15 \\
\hline Range & $8-25$ & $11-21$ & $8-25$ \\
\hline \multicolumn{4}{|l|}{ LGD operative time [min]: } \\
\hline Median & 70 & 66 & 70 \\
\hline Range & $48-86$ & $58-74$ & $48-86$ \\
\hline \multicolumn{4}{|l|}{ Esophagectomy operative time [min]: } \\
\hline Median & 448 & 441.5 & 486 \\
\hline Range & $406-800$ & $435-448$ & $406-800$ \\
\hline \multicolumn{4}{|l|}{ Type of anastomosis: } \\
\hline Hand-sewn & $3(27)$ & 1 & 2 \\
\hline Stapled & $8(73)$ & 1 & 7 \\
\hline \multicolumn{4}{|l|}{ Length of ICU stay [days]: } \\
\hline Median & 4 & 5 & 3 \\
\hline Range & $2-23$ & $4-6$ & $2-23$ \\
\hline Median length of hospital stay [days]: & & & 20 \\
\hline Median & 24 & 21 & \\
\hline Range & $10-63$ & $10-32$ & $11-63$ \\
\hline
\end{tabular}

tomy. This was identified intra-operatively and managed using manual pressure only. All patients were scheduled for routine admission to the intensive care unit (ICU) post oesophagectomy. The median ICU stay was 4 (2-23) days, and the median overall hospital stay was 24 (10-63) days. All patients were discharged to their homes.

\section{Outcomes}

Two (18\%) patients developed anastomotic leak. Secondary outcomes are summarized in Table III. There were no inpatient mortalities. No patient required reoperation during the same admission. In 1 (9\%) patient the Penrose drain eroded into the gastric conduit distal to the anastomosis, and this patient subsequently developed an anastomotic stricture requiring endoscopic dilatation. There were no readmissions within 30 days of the oesophagectomy.

\section{Discussion}

The key finding of this study is that the risk of anastomotic leak is $18 \%$ following a hybrid McKe- own oesophagectomy with prior LGD. This is similar to other published data [1, 2, 23] on anastomotic leaks following McKeown oesophagectomy without a prior LGD. An earlier review [24] reported an anastomotic leak rate of up to $25 \%$ for a cervical anastomosis, but a more recent review article reported a rate of around 15\% [2]. Contemporary studies [23, 25] comparing post-operative outcomes between minimally invasive Ivor-Lewis and Mckeown approaches demonstrated leak rates of $5-6 \%$ for a cervical anastomosis at high-volume centres.

Factors related to ischaemic conditioning have been described in order to identify the specific process by which anastomotic leaks may be reduced. These include the decision to perform LGD, the extent of devascularization, and the optimal interval between LGD and oesophagectomy.

\section{LGD vs. none}

It has been hypothesized that performing an LGD procedure prior to an oesophagectomy allows for increased perfusion to the conduit through the development of a collateral blood supply to the future 
Table III. Post-operative complications after McKeown oesophagectomy in patients who underwent prior laparoscopic gastric devascularization, categorized by the detection of anastomotic leak on oesophagram

\begin{tabular}{|c|c|c|c|}
\hline Outcome & $\begin{array}{c}\text { Total }(n=11) \\
n(\%)\end{array}$ & $\begin{array}{c}\text { Leak }(n=2) \\
n\end{array}$ & $\begin{array}{c}\text { No leak }(n=9) \\
n\end{array}$ \\
\hline Reoperation & $0(0)$ & 0 & 0 \\
\hline Inpatient mortality & $0(0)$ & 0 & 0 \\
\hline 30-day readmission & $0(0)$ & 0 & 0 \\
\hline Anastomotic stricture & $1(9)$ & 0 & 1 \\
\hline Pneumonia & $3(27)$ & 0 & 3 \\
\hline Respiratory failure & $1(9)$ & 0 & 1 \\
\hline Empyema & $0(0)$ & 0 & 0 \\
\hline Surgical site infection & $3(27)$ & 1 & 2 \\
\hline Chyle leak & $1(9)$ & 0 & 1 \\
\hline Recurrent laryngeal nerve injury & $1(9)$ & 0 & 1 \\
\hline Venous thromboembolic event & $1(9)$ & 0 & 1 \\
\hline
\end{tabular}

neo-oesophagus [9, 21, 26-28]. Studies performed in rats and pigs have demonstrated increased blood flow through the remaining arteries [29-32]. It is postulated that increased gastric conduit perfusion is through microcirculatory changes within the stomach. In human subjects, Pham et al. [12] studied the effects of ischaemic conditioning versus no ischaemic conditioning prior to oesophagectomy and demonstrated an increase in neovascularization at the microscopic level in those undergoing ischemic conditioning. Prudius et al. [33] corroborated these findings when investigating neovascularisation of human tissue samples after LGD. They identified a 3 -fold increase in the number of vessels of the resected stomach during oesophagectomy compared to tissue from resected stomachs of patients undergoing sleeve gastrectomy that served as controls.

Nguyen et al. [34] retrospectively looked at 81 oesophagectomy patients who underwent prior LGD versus 71 who did not and was unable to demonstrate a difference in postoperative leaks or strictures. This patient population, however, was a heterogenous mix of minimally invasive Ivor-Lewis, McKeown, and trans-hiatal oesophagectomies, without subgroup analysis. Thus, it is unknown whether the risk of developing a post-operative leak varies for different surgical approaches.

A systematic review and meta-analysis [35] failed to demonstrate reduction in the rate of anastomotic leak with gastric devascularization prior to oesophagectomy with an intrathoracic anastomo- sis; however, the studies selected for inclusion were all single-centre case series. Furthermore, there was heterogeneity with respect to the definition of anastomotic leak, the method of gastric devascularization, and the conditioning time. The authors performed subgroup analyses comparing 2 different types of conditioning (ligation versus embolization) and 2 different conditioning times ( $<2$ vs. $>2$ weeks) to controls and did not find a difference in anastomotic leak rates. However, they did report that those who underwent ischaemic conditioning had fewer postoperative complications and a lower likelihood of reoperation compared to those without conditioning.

A randomized controlled trial by Veeramootoo [36] compared no gastric devascularisation and oesophagectomy with left gastric artery ligation, and oesophagectomy 2 weeks later did not demonstrate a difference in gastric tip perfusion between the 2 groups. They concluded this was due to undetectable microcellular changes within the stomach.

Our data also did not demonstrate differences in rates of anastomotic leak after LGD followed by hybrid McKeown oesophagectomy compared to a hybrid McKeown oesophagectomy alone. There are a few possible explanations for this. Firstly, the 2 main causes for anastomotic leaks are poor blood supply and increased tension; ischaemic conditioning of the stomach offsets only the former. Microcirculatory changes and collateral vessel development may result in better perfusion of the gastric conduit tip at the time of oesophageal resection and anas- 
tomosis creation, but if the anastomosis is still under mechanical tension, then this enhanced blood flow may no longer protect against an anastomotic leak. Mckeown oesophagectomy differs from the more commonly performed Ivor Lewis oesophagectomy in that the gastric conduit has a further distance to travel to reach the neck. This is in contrast to Ivor-Lewis oesophagectomy, in which the conduit has less distance to travel and therefore may be under less tension.

Secondly, our study also differs from others by its hybrid approach to the oesophagectomy. Prior studies examined the difference with and without ischaemic conditioning of the stomach in minimally invasive Mckeown oesophagectomy. We begin with a thoracoscopic approach to mobilize the thoracic oesophagus, followed by an upper midline laparotomy to create the gastric conduit, resection of the oesophagus, and creation of the oesophagogastric anastomosis through a left cervical incision. Our findings may not be directly comparable to those of a minimally invasive approach because a laparotomy affords haptic feedback that is not present with laparoscopy. By manually pulling the gastric conduit through the mediastinum, the operating surgeon can identify excess tension and correct this before the anastomosis is created.

\section{Extent of devascularization}

Few studies have investigated the effect of a greater extent of devascularization in humans. Pham et al. [21] did not demonstrate a statistically significant difference in clinical anastomotic leaks when they compared partial (division of only the short gastric vessels) versus complete (division of left gastric vessels and short gastric vessels) ischaemic conditioning of the stomach prior to a minimally invasive oesophagectomy. Other groups have explored this phenomenon in animal models. For instance, Jones Beck et al. [28] found that ligation of both the left gastric artery and short gastric arteries during laparoscopy 3 weeks prior to oesophagectomy resulted in improved blood flow at both the proximal and distal stomach during a trans-hiatal oesophagectomy in mongrel dogs when compared to ligation of short gastric arteries alone, although this was not statistically significant $(p=0.07)$.

Our patient population underwent ischaemic conditioning of the stomach by laparoscopic ligation of all vessels supplying the stomach except the right gastroepiploic and right gastric vessels. We speculate that a further reason our data does not demonstrate reduction in anastomotic leak compared to that reported in the literature is because although significant changes in microcirculation following ischaemic conditioning have been reported in animal studies, the clinical importance of this is not yet clear, and there may be other factors not accounted for that explain the reduction in the number of anastomotic leaks.

\section{Ischaemic conditioning time}

Regarding the ischaemic conditioning time (the interval between LGD and oesophagectomy), Veeramootoo et al. [37] were able to demonstrate a time-dependent influence of ischaemic conditioning of the stomach. They showed a reduction in the rate of anastomotic leak from 100\% (7 out of 7 patients) to 5.7\% (2 out of 35 patients) with LGD performed 5 days versus 2 weeks prior to an Ivor-Lewis oesophagectomy. Perry et al. [38] investigated the rates of anastomotic leaks and strictures in patients who underwent a LGD either 1 or 12 weeks prior to minimally invasive McKeown oesophagectomy. They reported no leaks in the 12-week group versus $14 \%$ in the 1 -week group, although these results did not achieve statistical significance. This group went on to study time intervals for ischaemic conditioning in American opossums [39] (ischaemic conditioning time of 7 days, 30 days, 90 days, and no gastric devascularization) and found that the animals in the 30-day group had fewer subclinical leaks, increased neovascularization, and decreased inflammation at the anastomotic site compared to the other 3 groups. Reavis et al. [40] demonstrated that 28 days were required for optimal perfusion at the gastric tip. Another study [33] noted greater neovascularisation in tissue samples at 90-140 days compared to 3045 days post LGD. However, all these studies had small sample sizes, and the results should be interpreted with caution.

The maximum conditioning time in our study was only 25 days, based on the logistics within our centre; therefore, the lack of reduction in anastomotic leak rates in our study may be due to the shorter time interval and the theoretical lack of sufficient circulatory change at the gastric fundus.

There are some limitations that warrant discussion. First, this is a single-centre study with a small cohort size in which it may be difficult to detect clinically significant changes. We did not have a control group, and our results are compared to other studies. Second, we did not have information on in- 
tra-operative use of vasopressors or blood transfusions, which may be potentially confounding factors. Third, we performed a handsewn anastomosis in 3 patients and a stapled anastomosis in the remainder. Thus, it is unclear in our series whether anastomotic technique was a factor in the development of post-operative anastomotic leak. Finally, we were not able to assess conduit perfusion intra-operatively. To our knowledge, no prior studies have investigated the change in perfusion at the tip of the gastric conduit during the pull-through to the neck, and this may provide the basis for future research.

\section{Conclusions}

Performing laparoscopic ischaemic conditioning of the stomach up to 4 weeks prior to hybrid minimally invasive McKeown oesophagectomy may not reduce the risk of anastomotic leak compared to the published literature on McKeown oesophagectomy alone. LGD may be an unnecessary procedure in the surgical management of resectable oesophageal cancer.

\section{Conflict of interest}

Drs. Hanna, Mir, Williams, Merchant and Chung declare no conflict of interest. Dr. Zevin holds a research grant from Medtronic and an educational grant from Ethicon that is unrelated to this study.

\section{References}

1. Markar SR, Arya S, Karthikesalingam A, Hanna GB. Technical factors that affect anastomotic integrity following esophagectomy: systematic review and meta-analysis. Ann Surg Oncol 2013; 20: 4274-81.

2. Mitchell JD. Anastomotic leak after esophagectomy. Thorac Surg Clin 2006; 16: 1-9.

3. Schuchert MJ, Abbas G, Nason KS, et al. Impact of anastomotic leak on outcomes after transhiatal esophagectomy. Surgery 2010; 148: 831-8.

4. Derogar M, Lagergren P. Health-related quality of life among 5-year survivors of esophageal cancer surgery: a prospective population-based study. J Clin Oncol 2012; 30: 413-8.

5. Urschel JD. Esophagogastric anastomotic leaks: the importance of gastric ischemia and therapeutic applications of gastric conditioning. J Invest Surg 1998; 11: 245-50.

6. Rissanen TT, Vajanto I, Hiltunen MO, et al. Expression of vascular endothelial growth factor and vascular endothelial growth factor receptor-2 (KDR/Flk-1) in ischemic skeletal muscle and its regeneration. Am J Pathol 2002; 160: 1393-403.

7. Schröder W, Stippel D, Gutschow C, et al. Postoperative recovery of microcirculation after gastric tube formation. Langenbeck's Arch Surg 2004; 389: 267-71.
8. Mittermair C, Klaus A, Scheidl S, et al. Functional capillary density in ischemic conditioning: implications for esophageal resection with the gastric conduit. Am J Surg 2008; 196: 88-92.

9. Bludau M, Hölscher AH, Vallböhmer D, et al. Ischemic conditioning of the gastric conduit prior to esophagectomy improves mucosal oxygen saturation. Ann Thorac Surg 2010; 90: 1121-6.

10. Bludau M, Hölscher AH, Vallböhmer D, et al. Vascular endothelial growth factor expression following ischemic conditioning of the gastric conduit. Dis Esophagus 2013; 26: 847-52.

11. Nishikawa K, Matsudaira H, Suzuki H, et al. Intraoperative thermal imaging in esophageal replacement: its use in the assessment of gastric tube viability. Surg Today 2006; 36: 802-6.

12. Pham TH, Perry KA, Enestvedt CK, et al. Decreased conduit perfusion measured by spectroscopy is associated with anastomotic complications. Ann Thorac Surg 2011; 91: 380-5.

13. Park SY, Kang WJ, Cho A, et al. 64CU-ATSM hypoxia positron emission tomography for detection of conduit ischemia in an experimental rat esophagectomy model. PLoS One 2015; 10: e0131083.

14. Fikfak V, Gaur P, Kim MP. Endoscopic evaluation of gastric conduit perfusion in minimally invasive Ivor Lewis esophagectomy. Int J Surg Case Rep 2016; 19: 112-4.

15. Liebermann-Meffert DMI, Meier R, Siewert JR. Vascular anatomy of the gastric tube used for esophageal reconstruction. Ann Thorac Surg 1992; 54: 1110-5.

16. Liebermann-Meffert D. Anatomical basis for the approach and extent of surgical treatment of esophageal cancer. Dis Esophagus 2001; 14: 81-4.

17. Nguyen NT, Longoria M, Sabio A, et al. Preoperative laparoscopic ligation of the left gastric vessels in preparation for esophagectomy. Ann Thorac Surg 2006; 81: 2318-20.

18. Hölscher AH, Schneider PM, Gutschow C, Schröder W. Laparoscopic ischemic conditioning of the stomach for esophageal replacement. Ann Surg 2007; 245: 241-6.

19. Varela E, Reavis KM, Hinojosa MW, Nguyen N. Laparoscopic gastric ischemic conditioning prior to esophagogastrectomy: technique and review. Surg Innov 2008; 15: 132-5.

20. Yuan Y, Duranceau A, Ferraro P, et al. Vascular conditioning of the stomach before esophageal reconstruction by gastric interposition. Dis Esophagus 2012; 25: 740-9.

21. Pham TH, Melton SD, McLaren PJ, et al. Laparoscopic ischemic conditioning of the stomach increases neovascularization of the gastric conduit in patients undergoing esophagectomy for cancer. J Surg Oncol 2017; 116: 391-7.

22. Strosberg DS, Merritt RE, Perry KA. Preventing anastomotic complications: early results of laparoscopic gastric devascularization two weeks prior to minimally invasive esophagectomy. Surg Endosc Other Interv Tech 2017; 31: 1371-5.

23. Brown AM, Pucci MJ, Berger AC, et al. A standardized comparison of peri-operative complications after minimally invasive esophagectomy: Ivor Lewis versus McKeown. Surg Endosc 2018; 32: 204-11.

24. Urshel JD. Ischemic conditioning of the rat stomach: implications for esophageal replacement with stomach. J Cardiovasc Surg 1995; 36: 191-3.

25. Luketich JD, Pennathur A, Awais O, et al. Outcomes after minimally invasive esophagectomy: review of over 1000 patients. Ann Surg 2012; 256: 95-103. 
26. Cuenca-Abente F, Assalia A, del Genio G, et al. Laparoscopic partial gastric transection and devascularization in order to enhance its flow. Ann Surg Innov Res 2008; 2: 3.

27. Diana M, Hbner M, Vuilleumier $H$, et al. Redistribution of gastric blood flow by embolization of gastric arteries before esophagectomy. Ann Thorac Surg 2011; 91: 1546-51.

28. Jones Beck SM, Malay MB, Gagné DJ, et al. Experimental model of laparoscopic gastric ischemic preconditioning prior to transhiatal esophagectomy. Surg Endosc Other Interv Tech 2011; 25: 2470-7.

29. Urschel JD. Esophagogastrostomy anastomotic leaks complicating esophagectomy: a review. Am J Surg 1995; 169: 634-40.

30. Urschel JD, Takita H, Antkowiak JG. The effect of ischemic conditioning on gastric wound healing in the rat: implications for esophageal replacement with stomach. J Cardiovasc Surg 1997; 38: 535-8.

31. Urschel JD, Antkowiak JG, Delacure MD, Takita H. Ischemic conditioning (Delay Phenomenon) improves esophagogastric anastomotic wound healing in the rat. J Surg Oncol 1997; 66: 254-6.

32. Gomes M, Ramacciotti E, Miranda F, et al. Vascular flow of the gastric fundus after arterial devascularization: an experimental study. J Surg Res 2009; 152: 128-34.

33. Prudius V, Procházka V, Pavlovský Z, et al. Neovascularization after ischemic conditioning of the stomach and the influence of follow-up neoadjuvant chemotherapy thereon. Videosurgery Miniinv 2018; 13: 299-305.

34. Nguyen NT, Nguyen XMT, Reavis KM, et al. Minimally invasive esophagectomy with and without gastric ischemic conditioning. Surg Endosc Other Interv Tech 2012; 26: 1637-41.

35. Heger P, Blank S, Diener MK, et al. Gastric preconditioning in advance of esophageal resection-systematic review and metaanalysis. J Gastrointest Surg 2017; 21: 1523-32.

36. Veeramootoo D, Shore AC, Wajed SA. Randomized controlled trial of laparoscopic gastric ischemic conditioning prior to minimally invasive esophagectomy, the LOGIC trial. Surg Endosc Other Interv Tech 2012; 26: 1822-9.

37. Veeramootoo D, Shore AC, Shields B, et al. Ischemic conditioning shows a time-dependant influence on the fate of the gastric conduit after minimally invasive esophagectomy. Surg Endosc Other Interv Tech 2010; 24: 1126-31.

38. Perry KA, Enestvedt CK, Pham TH, et al. Esophageal replacement following gastric devascularization is safe, feasible, and may decrease anastomotic complications. J Gastrointest Surg 2010; 14: 1069-73.

39. Perry KA, Banarjee A, Liu J, et al. Gastric ischemic conditioning increases neovascularization and reduces inflammation and fibrosis during gastroesophageal anastomotic healing. Surg Endosc Other Interv Tech 2013; 27: 753-60.

40. Reavis KM, Chang EY, Hunter JG, et al. Utilization of the delay phenomenon improves blood flow and reduces collagen deposition in esophagogastric anastomoses. Ann Surg 2005; 24: 736-45.

Received: 3.01.2021, accepted: 3.02.2021. 